\title{
Caracterización de la diáspora de talentos venezolanos
}

\section{Characterization of the Venezuelan talent diaspora}

\author{
MARTINEZ, José M. ${ }^{1}$
}

\section{Resumen}

En este trabajo se discuten las características de la diáspora actual de talentos venezolanos de alta calificación, según se han manifestado mediante una encuesta realizada a fines de 2019. Se muestra el alto nivel de calificación profesional de científicos, profesores y profesionales que han emigrado desde hace muchos años, así como más recientemente. Se destacan sus características demográficas, los numerosos títulos universitarios, su dominio del inglés y otras altas capacidades personales. Se presentan también las dificultades que han encontrado para conseguir acceso a trabajos correspondientes a sus capacidades intelectuales y sus expectativas en relación a su posible contribución para la reconstrucción de Venezuela.

Palabras clave: talentos, migraciones, éxodo de personas cualificadas, diáspora venezolana

\begin{abstract}
This paper discusses the characteristics of the current diaspora of highly qualified Venezuelan talents, as shown by a survey carried out at the end of 2019. The high level of professional qualification of scientists, teachers and professionals who have emigrated many years ago as well as more recently is shown. Their demographic characteristics, numerous university degrees, their command of English and other high personal abilities are highlighted. The difficulties they have encountered in gaining access to jobs corresponding to their intellectual capacities, and their expectations regarding their possible contribution to the reconstruction of Venezuela are also presented.

Key words: talents, migrations, exodus of qualified people, Venezuelan diaspora
\end{abstract}

\section{Introducción}

La problemática de la diáspora de venezolanos por todo el mundo ha seguido aumentando ante el gran número de emigrados, cuyas últimas cifras alcanzan ya a cerca de los seis millones, entre las cuales se puede estimar que hay muchísimos profesionales universitarios,. Se encuentran talentos de todas las profesiones, sobre todo médicos, odontólogos, ingenieros, profesores y científicos.

Las condiciones de vida en Venezuela se han ido empeorando desde 2014. La aplicación por veinte años de políticas públicas ineficaces ha acarreado altos índices de pobreza, inflación, escasez de productos de primera necesidad (alimentos y medicinas), los mayores niveles de inseguridad del mundo y un país quebrado técnica y económicamente. Como resultado Venezuela ha experimentado la destrucción y retroceso en todos los ámbitos que definen a un país. La aplicación sostenida de un modelo anacrónico que busca generar un control hegemónico ha convertido a Venezuela en un país emisor de millones de personas. Dentro de ese contingente, según De la Vega (2017), existe un porcentaje significativo de profesionales científicos y académicos,

\footnotetext{
${ }^{1}$ Universidad Central de Venezuela. Email: jmmartinezcabrero@gmail.com
} 
categorizados como éxodo de competencias. La mayor concentración de emigración profesional e intelectual venezolana está ubicada en Estados Unidos, algunos países de América Latina y Europa.

Ante esta situación se decidió iniciar una investigación sobre las condiciones en que se encuentran los talentos que han emigrado en los últimos años mediante la gestión de una encuesta. También se solicitó a un grupo de talentos que emigraron hace tiempo y a otros que lo hicieron recientemente que relatasen cómo se las han arreglado en ese proceso de emigración. Los resultados de la encuesta, otras informaciones y artículos escritos por estudiosos venezolanos sobre la problemática de las migraciones han sido publicados en un libro.(Martínez, 2021)

\subsection{Caracterización de la diáspora de venezolanos de alta calificación}

Eventos, decisiones y políticas de los dos últimos gobiernos en Venezuela, inciden en el aumento de emigración del talento científico, académico y profesional. Claudio Bifano destaca que el actual régimen político ${ }^{2}$

"En todos los niveles del establecimiento científico nacional, profesionales sin
experiencia han sido asignados (con poco conocimiento científico o técnico o
antecedentes) a puestos de autoridad o envergadura. Han sido elegidos sobre la base
de su lealtad al partido político en el poder", "las universidades y los centros de
investigación han sido objeto de drásticos recortes presupuestarios, afectando
gravemente la mayoría de programas de investigación actuales. La pérdida de capital
intelectual, emigrando a países como Estados Unidos, Canadá, México, Brasil, Francia,
España y otros países, se han acelerado. Jóvenes profesionales, académicos, científicos,
expertos en tecnología, médicos, e ingenieros están saliendo del país". (Bifano, 2009,
p. 1514)

En otros países pueden verse ejemplos exitosos en la gestión de la diáspora del talento profesional y científico, que incluyen la coordinación desde la llegada del capital humano al país de acogida, su inserción en la sociedad (idioma, vivienda, familia, empleo, etc.) y su intercambio con el mundo profesional, académico y científico del país de acogida. Se prevé no sólo la preservación en la formación y experiencia del profesional, académico o científico, sino que además permite la geolocalización y vinculación del recurso humano al país de origen. (TWAS, 2018).

La forma de migración del talento venezolano es distinta a la de otras formas de migración. Es silenciosa. La mayoría se desplaza utilizando sus propios recursos económicos. De hecho, la diáspora del talento - éxodo de intelectuales o científicos, académicos y profesionales emigrados o en el exilio - no entra en programas de políticas públicas. A esto se añade la ausencia de esfuerzos, por parte de las agencias gubernamentales en países de acogida, de una adecuada clasificación de la persona inmigrante de acuerdo al nivel de instrucción, universitario, etc.

Muchos profesionales, académicos y científicos venezolanos luchan en sus nuevos países de forma individual y sin ninguna clase de apoyo u orientación. La información de posibles programas para científicos, profesionales exiliados está dispersa y fragmentada y varía ampliamente de un país a otro. Adicionalmente, los programas impulsados para científicos y profesionales en el exilio, creados por los países de acogida, están orientados a países vecinos. Venezuela no se encuentra en la lista de países participantes en estos programas. Así mismo, en

2 Se denomina régimen político al conjunto de instituciones que regulan la lucha por el poder y su ejercicio, y de los valores que sustentan esas instituciones. http://www.eumed.net/diccionario/definicion.php?dic=3\&def=461 
los países de acogida no existe actualmente un mecanismo para (en función de los aciertos y desaciertos de programas o políticas previas) mejorar y rediseñar programas que tengan una mayor amplitud. (TWAS, 2017)

Como consecuencia de la desatención a la diáspora del talento, muchos científicos, profesionales y académicos venezolanos, experimentan altos niveles de desempleo y sus habilidades están significativamente subutilizadas, con frecuencia están subempleados en trabajos poco calificados y temporales o mal remunerados. Por tanto, sus posibilidades de volver a las carreras profesionales a menudo disminuyen a medida que permanecen desempleados en el nuevo país de residencia, ya que pierden la calificación y pierden autoestima.

En los años '70 y '80 en Venezuela se llevó a cabo el programa Gran Mariscal de Ayacucho para facilitar la formación en el extranjero de jóvenes con buen desempeño estudiantil. Actualmente no se puede asegurar que exista en Venezuela algún programa internacional para mejorar la formación de investigadores, profesores y profesionales, ni para encargarse del conocimiento que emigra o ha emigrado, ni para gestionar el éxodo intelectual. Tampoco existen estrategias y programas eficaces que posibiliten la incorporación efectiva profesionalmente en los países de acogida y la futura adecuada contribución del recurso humano emigrado en el marco de la reconstrucción del país, del desarrollo socio-económico, de la investigación, el desarrollo tecnológico y la innovación (I+D+i), tan necesarios para su rescate. (Kerdel-Vegas, 2000, p. 78)

En Estrada (2019) se plantea que el perfil de muchos emigrantes venezolanos es de alta calificación y que esta mayoría de académicos y profesionales con estudios universitarios preferirían seguramente poder aplicar y desarrollar sus capacidades intelectuales.

Un estudio realizado en Colombia en 2018 indicaba que, de los 202.046 migrantes legalizados (con Permiso Especial de Permanencia-PEP) (de los 442.462 elegibles en un total estimado en 1.032 .016 migrantes provenientes de Venezuela para el 30/9/2018) un $12,9 \%$ de ellos tenía títulos universitarios y un $0,4 \%$ postgrados, lo cual da unas cifras totales de 26.872 con estudios universitarios y de 8.081 con postgrado. (Bahar, 2018, p. F273)

\section{Metodología}

El primer desafío fue obtener directamente información referente a: ¿quiénes son los científicos, profesionales y académicos venezolanos que han emigrado?, ¿dónde están?, ¿cuál es su experiencia?, ¿cuáles son sus expectativas?. Siempre se consideró que es necesario crear una base de datos que contenga información sobre científicos y profesionales emigrados, refugiados, exiliados o desplazados (habilidades, experiencia, nivel de formación y ubicación geográfica), indispensable para la gestión eficiente de la preservación del talento intelectual y profesional, su sana integración profesional en los países de acogida y su vinculación para la reconstrucción del país.

Ahora bien, la dispersión geográfica internacional en que están actualmente los venezolanos se ha realizado por iniciativas individuales, sin que existan programas que los hayan agrupado y que permitan disponer de información accesible. Ante estas circunstancias se utilizó el método bola de nieve, haciendo también convocatorias por las redes sociales más consultadas por los profesionales, especialmente Linkedin. Se les pidió que distribuyesen el mensaje a sus conocidos. Los detalles del contenido de la encuesta se presentan en el anexo 5.1.

Para contribuir a la difusión del conocimiento sobre los talentos venezolanos, se consideró que es necesario y urgente poner en práctica programas que den a conocer internacionalmente sus características, informar de su nivel profesional, de cómo se han formado, cuáles son sus capacidades y cuáles son las dificultades que están encontrando para poder hacer uso de éstas, para conseguir trabajo remunerado estable, así como para que 
puedan tener un mayor desarrollo profesional, utilizando sus conocimientos, mejorándolos y haciendo aportes en los países donde están viviendo ahora.

Con este objetivo se realizó una encuesta amplia que fue realizada entre el 1 de Octubre de 2019 y el 31 de diciembre de 2019. Fue contestada en el plazo de esos tres meses por 95 profesionales. Los principales resultados se muestran más adelante, en la Sección 3.

Los conocimientos aportados por este trabajo podrán ser utilizados para:

- Facilitar el conocimiento de la problemática de la migración de talentos venezolanos en los países receptores y de las capacidades de éstos. Se podrá facilitar su incorporación a organizaciones locales, desarrollando actividades profesionales y perfeccionando sus conocimientos y destrezas.

- Articular la capacidad profesional de los talentos emigrados para aprovecharla en el futuro desarrollo de una nueva Venezuela.

- Articular a los talentos que están en Venezuela con los emigrados para aprovechar las experiencias de los países más adelantados en el campo de la ciencia y la tecnología.

- Canalizar los estudios y reflexiones sobre la problemática del éxodo de talentos en Venezuela.

- Identificar y gestionar la creación de recursos económicos para llevar adelante este proyecto y para mantener una gestión continua y regular de contacto e interacción con los talentos venezolanos que queden en otros países

\subsection{Características de la encuesta}

Tal como se ha mencionado, la encuesta está dirigida a los talentos emigrados, académicos y profesionales, y tiene como finalidad ir elaborando una base de datos estadística que permita dar a conocer sus características e intereses. Se considera que la diáspora del talento venezolano es una fuerza que difunde no solo el conocimiento, sino también la tolerancia y el entendimiento intercultural y que puede y debe contribuir a la recuperación de Venezuela, pudiendo contribuir con el desarrollo del conocimiento en los países de acogida.

Se ha tenido mucho cuidado al solicitar los datos para que se proteja la privacidad de los encuestados. Por eso se siguieron las pautas establecidas por la Unión Europea para el tratamiento de datos personales sensibles (Artículo 13 de la regulación (UE) 2016/679). El contenido final de la encuesta fue depurado consultando a diversas personas interesadas en la materia para suprimir preguntas personales que realmente no agregasen valor a los objetivos de la encuesta. Algunos datos solicitados son obligatorios y otros son opcionales. Se asegura que los datos personales se utilizarán solamente para estudios estadísticos y que si se desean utilizar con otra finalidad se les pedirá permiso. Se pide el número de teléfono (opcional) y el correo, para poder establecer contacto con ellos individualmente cuando fuere conveniente.

Se solicitan inicialmente ciertos datos personales, tales como: Nombre, Apellidos, País de nacimiento, Año de nacimiento. Nacionalidad de nacimiento, Segunda nacionalidad, y Sexo.

Con estos datos se busca caracterizar la edad, sexo, su origen y si tienen una segunda nacionalidad que les facilite la residencia en los países a los que se han trasladado. Se les pide también en qué país y ciudad viven para conocer la variedad de geolocalización en que se encuentran.

La información sobre los títulos que tienen permite conocer hasta que nivel académico han llegado, y también información sobre el tipo de actividades que venían realizando en Venezuela (entre ellas: investigación, docencia, ejercicio profesional en empresas públicas o privadas, consultoría, participación en ONG, o trabajos como emprendedores o empresarios). Estas actividades, que pueden haberse desarrollado simultáneamente o en épocas distintas, ofrecen un conocimiento interesante cuando se analicen, para saber hasta qué punto nuestros talentos se concentran en una sola actividad o son abiertos a múltiples facetas en su ejercicio profesional. 
Otras preguntas indagan acerca de sus actividades actuales para ver su correspondencia con las que venían desarrollando.

El dominio de los idiomas es hoy día un factor clave para defenderse profesionalmente, sobre todo cuando muchos de los migrantes se han dirigido hacia los Estados Unidos o a países europeos. También una proporción importante se han dirigido a países donde se habla en castellano (España y países de América Latina). La localización permitirá ver hasta qué punto puede haber influido la facilidad para comunicarse con la selección de país al que emigrar. Se puede así establecer relación entre los países en que viven y el dominio de otros idiomas para conocer, si la falta de conocimiento del idioma del país en que están, puede o no ser un factor que influya en su incorporación profesional.

La encuesta trata también de obtener información sobre los planes futuros de los encuestados acerca de su disposición a regresar a Venezuela cuando las condiciones actuales cambien o a quedarse donde viven ahora. También sobre su disposición a participar en la reconstrucción de Venezuela, y las áreas en que creen que puede ser útil.

Finalmente se hacen algunas preguntas acerca de su experiencia como migrantes: su integración al país donde viven, el tiempo que hace que salieron de Venezuela, las posibilidades y dificultades para conseguir trabajo y el valor que ha tenido su calificación y experiencia para obtenerlo.

\section{Resultados y discusión}

Unos primeros resultados de la encuesta, indican que existe una gran variedad de respuestas.

De los noventa y cinco (95) participantes, $62 \%$ de los encuestados son de sexo masculino.

En cuanto a edad, la edad promedio es de 52 años y va desde 26 hasta 85 años, con una distribución bastante uniforme, diecisiete (17) son menores de treinta y cinco (35) años, el 18\%. Esto muestra que los más jóvenes, entre veinte (20) y treinta y cinco (35) años muestran ya su importancia en la emigración.

La gran mayoría, ochenta y cuatro personas que respondieron la encuesta nacieron en Venezuela (89\%) y cuatro en España(6\%). Esto muestra el peso de los nacidos en Venezuela en el fenómeno de la diáspora de talentos. Hay que agregar que los nacidos en España incluye a hijos de españoles emigrados, nacidos en España, que se fueron a Venezuela como consecuencia de la guerra civil y ante la apertura a las migraciones para reclutar trabajadores. Muchos se formaron profesionalmente en Venezuela y ahora realizan una nueva emigración.

Casi la mitad tienen doble nacionalidad, $13 \%$ han adquirido la nacionalidad española, $10 \%$ la italiana y $4 \%$ la de Estados Unidos y en un $12 \%$ la segunda nacionalidad es venezolana, lo cual muestra lo que se dijo antes, que parte de esta migración está compuesta por emigrantes que llegaron a Venezuela en la época en que la inmigración de europeos estuvo abierta (años '50 a '70). 8\% manifestaron tener una segunda nacionalidad distinta a las anteriores, el país de residencia de estas personas identifica a Francia, Alemania, Argentina, Canadá y México. Estos datos muestran el interés de las personas por incorporarse a los países de acogida, seguramente para facilitar sus posibilidades de trabajo y eventualmente porque ya decidieron quedarse definitivamente en esos países.

La lengua materna del $96,8 \%$ es el castellano

La mitad indican que tienen un nivel avanzado en inglés, pero prácticamente todos tienen nivel básico, intermedio o avanzado. Esto muestra que los talentos venezolanos encuestados están bien preparados para actuar en otros países Otros segundos idiomas señalados son el francés y el italiano en los cuales una cuarta parte dicen tener un conocimiento básico, pero la mitad dicen no tenerlo en absoluto. Algunos incluyen el 
alemán, el japonés básico y hablado, el ruso, el latín y el griego moderno. También se mencionan catalán, euskera y gallego, lo cual indica la adaptación a las regiones de España donde se encuentran.

La mayoría de los talentos venezolanos que están en la diáspora obtuvieron sus primeros títulos universitarios en dieciséis (16) universidades venezolanas, pero también siete indican que se graduaron la primera vez en el extranjero (Estados Unidos, Francia e Italia). Más de la mitad de los encuestados han egresado de las principales universidad de Caracas, (UCV, USB y UCAB), pero se mencionan otras 19 universidades, de las cuales 7 son de países extranjeros (Estados Unidos, Italia, Francia).

A pesar del posible sesgo de las respuestas debido a la metodología usada, la gran dispersión de los sitios de destino a los cuales han llegado los encuestados deja ver la esencia de la diáspora. A pesar del pequeño tamaño de esta muestra respecto al numero de talentos venezolanos emigrados, ya pueden identificarse diecisiete (17) países diferentes en los que residen los encuestados, ubicados en Europa, Norte y Suramérica. Una tercera parte de estos están en España, país donde se inició la encuesta.

Acerca del tiempo que los encuestados tienen fuera de Venezuela, el tiempo promedio fuera de Venezuela es de sesenta y ocho (68) meses, o sea cinco años y ocho meses. Por debajo de ese promedio se encuentran sesenta y cuatro (64) personas, o sea un $70 \%$ de los noventa y dos que respondieron esta pregunta. Solamente siete (7) personas indicaron que tienen más de veinte (20) años desde que salieron de Venezuela, o sea antes de la llegada al poder de Hugo Chávez Frías, estos talentos se estabilizaron en los países desarrollados y pudieron desarrollar sus carreras profesionales debido a sus altas capacidades. Más de la mitad tienen tres o menos años desde que emigraron, corresponidendo a la época de mayores dificultades de vida en Venezuela.

Entre noventa y dos (92) encuestados, se dan treinta y ocho (38) títulos diferentes entre los cuales destaca casi la mitad en las áreas científico-técnicas: por una parte treinta (30) ingenieros y quince (15) egresados en carreras científicas (Física, Química, Matemáticas, Computación). Debe destacarse también que veinte de los encuestados indican que tienen diecisiete títulos adicionales, algunos títulos universitarios y sobre todo especializaciones en asuntos administrativos, como gerencia de proyectos, finanzas, mercadeo.

Cuarenta y siete (47) personas tienen títulos de master, magister o DEA y treinta (30) tienen PhD o doctorado en veintisiete (27) especialidades distintas.

En relación con la búsqueda de trabajo, las tres cuartas partes de los encuestados han buscado trabajo, pero solamente la mitad lo ha hecho de manera estable. Treinta y seis por ciento (36\%) no ha conseguido trabajo en su especialidad. Cerca del ochenta por ciento (80\%) consideraron medianamente difícil, difícil o muy difícil conseguir trabajo. La mayor dificultad señalada por cerca del veinte por ciento (20\%) fue la edad. Otra dificultad señalada por la tercera parte de los encuestados es la falta de reconocimiento de su experiencia. También se mencionan: la falta de papeles de identificación y residencia, el reconocimiento, homologación y equivalencia de los títulos, la falta de experiencia, la falta de conocimiento del mercado laboral y la falta de contactos y redes profesionales.

Casi la mitad están dispuestos a regresar a Venezuela si la situación cambia, pero la cuarta parte preferiría quedarse definitivamente donde viven ahora.

Prácticamente el cien por ciento (100\%) están dispuestos a colaborar para el desarrollo de Venezuela.

El valor de esta pequeña encuesta comparada con el numeroso tamaño de la migración de profesionales universitarios puede apreciarse por la diversidad de las respuestas. A pesar de lo limitada y de no haber podido utilizar un método estadístico formal los resultados son altamente significativos. Se demuestra el alto nivel profesional de los encuestados, a pesar de la variedad de edades y tiemplo de emigración. Se señalan también algunas de las dificultades que encuentran para poder conseguir un trabajo profesional estable. 


\section{Conclusiones}

El éxodo de científicos, profesores y profesionales venezolanos ha seguido aumentando debido a las lamentables condiciones de crisis compleja que sufre Venezuela, existiendo una dispersión en muchos países de América Latina, Estados Unidos y Europa, sin que encuentren programas preparados para ayudarles a conseguir trabajo en sus profesiones.

Progresivamente se han ido poniendo en práctica iniciativas organizativas, mediante la creación de redes, que tratan de facilitar el contacto entre los distintos grupos de talentos emigrados, divulgar lo que está sucediendo en Venezuela y el conocimiento de sus competencias. Con estas informaciones se busca poder estimular a las instituciones en los países de acogida a que les ayuden a mantener un desarrollo profesional.

La encuesta muestra que hay una gran diversidad de personas y especialidades, que tienen un alto nivel de preparación y competencias profesionales y que los nuevos emigrados están haciendo esfuerzos para adaptarse a las nuevas condiciones, que muchos de ellos piensan regresar y que prácticamente todos desean contribuir al futuro desarrollo de Venezuela.

\section{Referencias bibliográficas}

Bahar, D., (2018). Migration, Knowledge Diffusion and the Comparative Advantage of Nations. The Economic Journal, 128(612), F273-F305. Recuperado de https://doi.org/10.1111/ecoj.12450.

Bifano. C. (2009): Venezuelan Science at Risk. Science, 324, p.1514.

De la Vega. I. (2017). Del éxodo de competencias a su geolocalización y vinculación. El caso Venezuela. En Bifano C. y Bonalde I. (Eds.). Planteamientos para una nueva visión de ciencia, tecnología y educación superior en Venezuela (437-470). Caracas: ACFIMAN- Colección Estudios.

Estrada, C. (2019). ¿Qué perfil tienen los venezolanos que emigran?, The conversation, 23 febrero 2019. Recuperado de: https://theconversation.com/que-perfil-tienen-los-venezolanos-que-emigran-112108.

Kerdel-Vegas, F. (2000). Diáspora del Talento. Programa Talven. Caracas: UNESCO-IESAL.

Martínez, J. M. (2021). Diáspora de talentos venezolanos: Características de una migración de alta calificación, Academia Nacional de la Ingeniería y el Hábitat, http://acading.org.ve/info/publicaciones/libros/pubdocs/Libro_Diaspora_de_talentos_venezolanos_Jose_ Manuel_Martinez.pdf

Páez, T. (2015). La voz de la diáspora, Madrid: Los libros de la catarata.

TWAS (2018). Accord provides opportunities for displaced scientists. The World Academy of Sciences (TWAS). News, 17 September 2018. Recuperado de: https://twas.org/article/accord-provides-opportunitiesdisplaced-scientists.

TWAS (2017). Refugee Scientists: Transnational Resources: Recommendations from a workshop, 13-17 March 2017, Trieste, Italy. The World Academy of Sciences (TWAS), Science policy. Recuperado de: https://twas.org/sites/default/files/twas_refugee_scientists_finalfinal.pdf. 


\section{Anexos}

\subsection{Encuesta CENSO PARA PROFESIONALES Y ACADÉMICOS VENEZOLANOS EMIGRADOS.}

Datos personales. * Requerido

1. Nombre*

2. Apellidos *

3. País de nacimiento

Mark only one oval.

- Venezuela

- España

- Estados Unidos

- Italia

- Portugal

- Otros país latinoamericano

- Otro país europeo

- Otros

4. Año de nacimiento *

\section{Nacionalidad}

5. Nacionalidad de nacimiento (nombre del país de su primera nacionalidad) * Mark only one oval.

○ Venezuela

○ España

- Italia

- Portugal

- Estados Unidos

- Otros

6. Segunda nacionalidad (nombre del país que le dio su segunda nacionalidad)* Mark only one oval.

- Venezuela

- España

- Italia

○ Portugal

- Estados Unidos

- Otros

- No tengo segunda nacionalidad

7. Sexo

Mark only one oval.

- Masculino

o Femenino

\section{Lugar de residencia actual *}

8. País

Mark only one oval.

- España

- Italia

- Francia

- Alemania

- Reino Unido

- Otro país europeo

- Estados Unidos

- Colombia

- Perú 


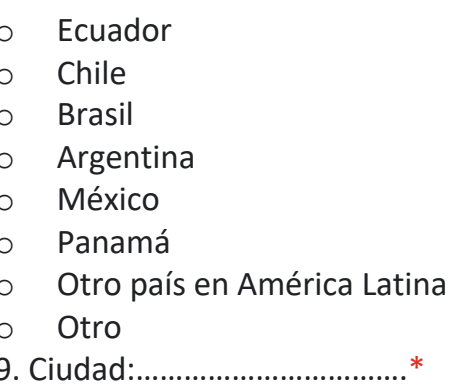

\section{Contacto}

Para el número telefónico por favor pongan + delante del código internacional y después el numero (Formato: + código internacional Número)

10. Teléfono personal móvil

11. Correo electrónico personal *

\section{Formación académica}

12. Título universitario inicial:.

13. Nombre de Universidad donde se graduó primera vez:

14. Otro título universitario:

15. Master/Magister (indicar especialidad):

16. Doctorado (Indicar especialidad):

17. Diplomados y otros títulos:

18. Otros estudios:......

\section{Áreas de actividad que ha tenido en Venezuela}

\section{Seleccione en todas en las que ha tenido actividades profesionales}

19. Área de actividad *

Check all that apply.

○ Investigación

- Docencia universitaria

- Consultoría

- Trabajo profesional en empresas privadas

- Trabajo profesional en empresas públicas

- Empresario/emprendedor

- Autónomo/Free Lancer

- ONG/asociaciones

- Empleado en administración pública

\section{Áreas de actividad actuales}

Seleccione en las que ha tenido actividades profesionales desde que salió de Venezuela

20. Área de actividad*

Check all that apply.

- Investigación

- Docencia universitaria

- Consultoría

- Trabajo profesional en empresas privadas

- Trabajo profesional en empresas públicas

- Empresario/emprendedor

- Autónomo/Free Lancer

- ONG/asociaciones

- Sin actividad profesional remunerada

- Empleado en administración pública 


\section{Áreas de especialidad en las que tiene más experiencia}

Indique como máximo las tres áreas en las que tiene importantes competencias

21. Área 1:

22. Área 2:

23. Área 3:

\section{¿A qué se dedica actualmente?}

Seleccione solamente aquella en la que tenga actualmente mayor dedicación

\section{Actividad *}

Mark only one oval.

- Investigación

- Trabajo profesional

- Docencia

- Empleado

- Autónomo

- Sin actividad remunerada

- Jubilado

\section{Idiomas}

Seleccionar solamente uno, el que corresponda como lengua materna, como primera lengua, los otros se indicarán en la pregunta siguiente

25. Lengua materna*

Mark only one oval.

- Castellano

- Inglés

- Francés

- Italiano

- Alemán

○ Portugués

- Otro

26. Otro idioma (indique cuál):

27. Otros idiomas *

Mark only one oval per row.

\begin{tabular}{|l|l|l|l|l|}
\hline & En absoluto & Básico & Intermedio & Avanzado \\
\hline Castellano & & & & \\
\hline Inglés & & & & \\
\hline Francés & & & & \\
\hline Italiano & & & & \\
\hline Alemán & & & & \\
\hline
\end{tabular}

28. Otros idiomas y nivel:.

\section{Opiniones}

¿Cómo visualiza su relación con Venezuela en el futuro?

29. ¿Qué planes futuros tiene? *

Check all that apply.

\begin{tabular}{|c|c|c|c|}
\hline \multirow{2}{*}{ Si cambia la situación política/social en Venezuela, ¿Regresaría Ud.? } & Sí & No & NS/NC \\
\hline & & & \\
\hline ¿Preferiría quedarse definitivamente donde vive ahora? & & & \\
\hline $\begin{array}{l}\text { ¿Está dispuesto a colaborar con el desarrollo productivo y el } \\
\text { crecimiento de Venezuela? }\end{array}$ & & & \\
\hline $\begin{array}{l}\text { ¿Mantiene contactos profesionales con instituciones de su área de } \\
\text { actividad? }\end{array}$ & & & \\
\hline
\end{tabular}

30. ¿En qué área, sector o campo de actividad le gustaría colaborar con las instituciones venezolanas? 


\section{Experiencia como emigrado}

¿Cómo se ha integrado profesionalmente en el país donde vive ahora?

31. ¿Hace cuántos meses que salió de Venezuela? *

32. ¿Qué le hizo seleccionar el país donde ha emigrado?:....

33. ¿Ha buscado trabajo en las áreas de su especialidad? *

Mark only one oval.

- Sí

- No

O Ocasionalmente

34. ¿Ha conseguido trabajo en las áreas de su especialidad? *

Mark only one oval.

○ Sí

- No

- Ocasionalmente

35. ¿Cuáles son las principales causas por las cuales no ha conseguido empleo en sus áreas de actividad?:

36. ¿Cómo evalúa el nivel de dificultad encontrado para conseguir trabajo?

Mark only one.

\begin{tabular}{|l|l|l|l|l|l|l|}
\hline & 1 & 2 & 3 & 4 & 5 & \\
\hline Muy baja & & & & & & Muy alta \\
\hline
\end{tabular}

37. ¿Está utilizando sus conocimientos y experiencia profesional en el trabajo que realiza?

Mark only one oval.

○ Sí

- No

- Parcialmente

38. ¿Ha tenido dificultades para conseguir que su experiencia haya sido reconocida?

Mark only one oval.

○ Sí

- No

- Parcialmente

Las informaciones aquí depositadas serán individualmente confidenciales y están protegidas por el tratamiento de datos sensibles personales a efecto del Artículo 13 de la Regulación (UE) 2016/679.

Al llenarlas uds. aceptan que se utilicen con fines estadísticos y que para cualquier otro uso deberán dar primero su autorización.

\section{(cc) BY-No}

Esta obra está bajo una Licencia Creative Commons Atribución-NoComercial 4.0 Internacional 